\title{
An Assessment of the Prevalence and Severity of Temporomandibular Disorders among Undergraduate Dental Students at Qassim University
}

\author{
Ahmed Ali Alfawzan
}

\begin{abstract}
Aim: Temporomandibular disorders (TMDs) represent disorders causing abnormality and impairment in the functionality of the temporomandibular joint (TMJ). The aim of this study was to assess the prevalence and severity of TMDs among the dental students of Qassim University using a Fonseca's questionnaire.

Material and methods: The Fonseca's questionnaire was distributed through university email to undergraduate dental students in Qassim University, and participants were briefed about the procedure for completing it. The questionnaire contained 10 questions that were related to the signs and symptoms of TMD. The subjects were instructed to answer each question with "yes," "no," or "sometimes." Scores were given, and the value was used to calculate the severity of TMD.

Results: The study showed that out of 200 students, 110 students (55\%) were found to have no TMD. Regarding the presence of TMD, about $45 \%$ of the students were found to have signs and symptoms, in which 33\% had mild TMD, 5\% had moderate TMD, and 7\% had severe TMD. The number of students who reported mild types of TMD (33\%) was significantly higher than those who reported moderate or severe types of TMD. Regarding the correlation of gender to the presence of TMD, $52 \%$ of female students showed some degree of TMD vs $42 \%$ of the male students. Conclusion: The present study shows that a mild-to-moderate prevalence of TMD appears to be evident among the dental students of Qassim University. Future research should, however, focus on longitudinal studies to identify and follow-up with TMD patients.

Clinical significance: This study highlighted the importance for the early diagnosis of the TMD and its relation to stress.

Keywords: Fonseca's questionnaire, Prevalence, Severity, Temporomandibular disorder.

World Journal of Dentistry (2020): 10.5005/jp-journals-10015-1715
\end{abstract}

\section{INTRODUCTION}

The research about temporomandibular joint (TMJ) function is ongoing, mainly because of the multifactorial etiology of temporomandibular dysfunction, which remains a cryptic issue among researchers. Temporomandibular disorder (TMD) is an umbrella term that defines a group of painful orofacial disorders which affects the TMJ or muscles of mastication. In some situations, it occurs in response to fatigue of the craniocervicofacial muscles. This fatigue leads to a limitation in the movement and function of the muscles and joint, which can become noticeable by an articular clicking sound.

Research has shown that roughly $33 \%$ of people have a TMD manifestation, and between $3.6 \%$ and $7 \%$ of people have severe TMD that requires medical attention. ${ }^{1}$ In a cross-sectional study conducted on Saudi children and adolescents, about $27.2 \%$ of the participants were diagnosed with at least one TMD sign or symptom. ${ }^{2}$ As a result of using assorted study designs as well as nonstandardized strategies to diagnose TMD, there are varying outcomes of reports of TMD prevalence from research studies. Temporomandibular disorder is a pervasive issue most seen in people from 20 to 40 years of age. According to researchers, its predominance ranges between 20 and $50 \%$ of this age group. ${ }^{3,4}$ The distinction in pervasiveness might be ascribed to contrasts in the ethnicity and race of participants, based on the sampling method. ${ }^{3}$

Temporomandibular disorder etiology is multifactorial. Important etiological factors include occlusal interferences, stress, tooth loss, postural changes, dysfunctions of the masticatory musculature, and intrinsic or extrinsic changes that affect the joint. ${ }^{5}$
Department of Preventive Dentistry, College of Dentistry, Qassim University, Ar Rass, Kingdom of Saudi Arabia

Corresponding Author: Ahmed Ali Alfawzan, Department of Preventive Dentistry, College of Dentistry, Qassim University, Ar Rass, Kingdom of Saudi Arabia, Phone: +966 553936688, e-mail: dr.ahmed. alfawzan@qudent.org

How to cite this article: Alfawzan AA. An Assessment of the Prevalence and Severity of Temporomandibular Disorders among Undergraduate Dental Students at Qassim University. World J Dent 2020;11(2):134-138. Source of support: Nil

Conflict of interest: None

The International Association for the Study of Pain refers to pain as sensory stimuli that leads to unpleasant emotional experiences. International Association for the Study of Pain argues that pain is a subjective experience and should not be directly linked to tissue damage. In other words, pain experience depends on the person experiencing the pain and not necessarily the presence of tissue damage. ${ }^{6}$

It is now well established that TMD is the main etiology of non-dental orofacial pain. The pain notably affects the masseter muscle, but pain can also be experienced in the preauricular region as well as in the anterior temporalis muscle area. ${ }^{7}$ The major signs of TMD, which can be observed in the opening and closing of the mouth, include muscle tenderness, deviation or deflection of the mandible, jaw lock, and the presence of clicking sounds. ${ }^{8}$ One 
significant symptom includes the presence of dull pain, which remains difficult to localize for the patient. In most cases, this occurs unilaterally. In some cases, the patient may experience sharp pain which deteriorates to a throbbing sensation. Experiencing stress, clenching teeth, and eating are important aggravating factors, while relaxing the muscles, applying heat, or taking an analgesic has proved to help reduce symptoms.

Like most diseases, early diagnosis and treatment of TMD are very important. Temporomandibular disorder is a progressive disease that gets worse with the passage of time. If it is treated in its early stages, then the prognosis is good. Although several TMD assessment tools have been proposed in literature, screening for TMDs from the population is still a huge challenge for researchers and clinicians. In 2014, Schiffman et al. ${ }^{9}$ proposed Research Diagnostic Criteria for TMDs, which are universally accepted as a tool to assess TMD. Research Diagnostic Criteria for TMDs was developed in the form of a questionnaire with high reliability and validity to screen pain related to TMD. A relevant questionnaire for assessing TMD is Fonseca's anamnestic index (FAI). Fonseca's anamnestic index has the advantages of being accessible to nonpatients, affordable, and easy to self-administer. It is a reliable tool to detect the signs and symptoms of TMD, thus making it useful in epidemiology and general practice. ${ }^{10}$

The Fonseca's questionnaire contains 10 questions designed to assess TMJ, head and back pain, and pain while chewing using a multidimensional approach. It also evaluates parafunctional habits, movement limitations, clicking sounds from joints, emotional stress, and malocclusion perception by participants.

The aim of the present study was to use an epidemiological survey to investigate the prevalence and severity of TMDs among the dental students of Qassim University using a Fonseca's questionnaire.

\section{Materials and Methods}

This descriptive cross-sectional study was approved by the Ethical Committee of the Dental College at Qassim University. Sample size was decided assuming the prevalence of TMDs to be $50 \%$. In total, 173 students will be appropriate to detect statistically significant difference of 5 and $80 \%$ power for analysis. This study was carried out between September 2019 and December 2019, and participants included undergraduate dental students of both genders studying in Qassim University. An electronic questionnaire was distributed to the students via university email as suggested by Fonseca (Table 1), and participants were given instructions for completing the questionnaire. The questionnaire was comprised of two parts. The first part contained questions about the participant's demographic information, medical and dental history, and history of major trauma to the face or head. The second part contained 10 questions related to the signs and symptoms of TMD, which included a survey of the presence of pain in the TMJ and head and back regions as well as several other parameters. The subjects were instructed to answer with "Yes," "No," or "Sometimes" and were not restricted by a time limit.

\section{Inclusion Criteria}

Undergraduate dental students from first to fifth year and interns of both genders studying in Qassim University.

\section{Exclusion Criteria}

Students with the history of major head or face trauma, orthodontic treatment, and/or musculoskeletal.

\begin{tabular}{ll} 
Table 1: & Fonseca's questionnaire \\
\hline S.no & Questions \\
\hline $1 \quad$ & $\begin{array}{l}\text { Is it hard for you to open your } \\
\text { mouth? }\end{array}$ \\
$2 \quad \begin{array}{l}\text { Is it hard for you to move your } \\
\text { mandible from side to side? }\end{array}$ \\
$3 \quad \begin{array}{l}\text { Do you get tired/muscular pain } \\
\text { while chewing? }\end{array}$ \\
$4 \quad \begin{array}{l}\text { Do you have frequent headaches? } \\
5\end{array}$ & $\begin{array}{l}\text { Do you have pain on the nape or } \\
\text { stiff neck? }\end{array}$ \\
6 & $\begin{array}{l}\text { Do you have earaches or pain in } \\
\text { craniomandibular joints? }\end{array}$ \\
7 & $\begin{array}{l}\text { Have you noticed any TMJ clicking } \\
\text { while chewing or when you open } \\
\text { your mouth? }\end{array}$ \\
9 & $\begin{array}{l}\text { Do you clench or grind your teeth? } \\
\text { Do your feel your teeth do not } \\
\text { articulate well? }\end{array}$ \\
$10 \quad \begin{array}{l}\text { Do you consider yourself a tense } \\
\text { (nervous) person? }\end{array}$
\end{tabular}

\section{Statistical Analysis}

The responses for the questionnaire were given scoring criteria. A score of " 10 " was given for every positive symptom, " 5 " was given for every occasional symptom, and " 0 " was given for the absence of symptoms. The sum of the scores of the individual questions was used to calculate the severity of TMD (Table 2). The severity of the disorder was classified into the categories "no TMD," "mild TMD," "moderate TMD," and "severe TMD." The analyses were performed using SPSS version 22.0 (IBM SPSS Statistics for Windows, Version 22.0; IBM Corp., Armonk, NY, USA). Pie charts, tables, and bar graphs were used to summarize the collected data.

\section{Results}

A total of 200 students participated in this study, including 142 males and 58 females, in which 36 students were from the first year, 35 from the second year, 34 students each from the third and fourth years, 33 from the fifth year, and 28 interns (Table 3). Among the group studied, 110 students (55\%) were found to have no TMD (Table 4). Of the participants who indicated the presence of TMD, of which $33 \%$ had mild TMD, $5 \%$ had moderate TMD, and $7 \%$ had severe TMD, about $45 \%$ were found to have signs and symptoms, including headache, jaw sound, neck pain, and limitations opening the mouth. The number of students with mild TMD was significantly higher than that of moderate and severe types of TMD (Fig. 1). Furthermore, $42 \%$ of the male students showed some degree of TMD vs $52 \%$ of the female students (Fig. 2). The symptom that received the highest rating of severity was reported to be frequent headaches (80.9\%), followed by neck pain (79.5\%), teeth clenching and grinding (76.3\%), clicking (69.6\%), difficulty opening the mouth $(66.5 \%)$, difficulty moving the mandible from side to side (61\%), muscle pain (49.3\%), and, finally, difficulty articulating the teeth (48\%) (Fig. 3).

\section{Discussion}

Temporomandibular disorder is a progressive disorder that has continually attracted researchers in search of etiological factors and 
Table 2: Clinical index classification-Fonseca

\begin{tabular}{ll}
\hline Score & Categories \\
\hline $0-15$ & No TMD \\
$20-40$ & Mild TMD \\
$45-65$ & Moderate TMD \\
$70-100$ & Severe TMD \\
\hline
\end{tabular}

Table 3: Year-wise distribution of the students

\begin{tabular}{ll}
\hline First year & 36 \\
Second year & 35 \\
Third year & 34 \\
Fourth year & 34 \\
Fifth year & 33 \\
Interns & 28 \\
\hline
\end{tabular}

Table 4: Sample characteristic, number, sex, and age group distribution

\begin{tabular}{lllllll}
\hline Fonseca's classification & Males $(n=142)$ & Percentage & Age group (years) & Females $(n=58)$ & Percentage & Age group $($ years $)$ \\
\hline Without signs and symptoms & 82 & 41 & $18-22$ & 28 & 14 & $19-23$ \\
With signs and symptoms & 60 & 30 & $20-25$ & 30 & 15 & $20-24$ \\
\hline
\end{tabular}

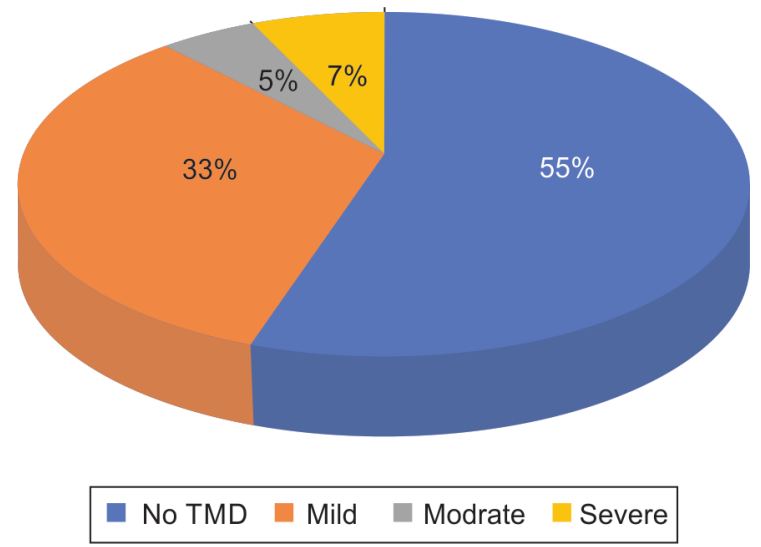

Fig. 1: Prevalence of temporomandibular disorders

the best management modalities to help patients impacted by the disorder. Previous studies indicate that early diagnosis is essential for the successful management of TMD, as the prognosis improves when interventions are provided at an early stage. ${ }^{1,2}$ Conversely, the prognosis becomes worse and irreversible at a later stage of diagnosis. One tool that has been proposed by previous researchers for early TMD detection is the Fonseca's questionnaire. ${ }^{10}$

The Fonseca's questionnaire is modeled on the anamnestic index that was initially developed together with other clinical indexes by Helkimo ${ }^{11}$ in 1974. These indexes were later adapted by Fonseca et al. in 1994 to form the Fonseca's questionnaire, or FAl, which give TMD the classifications ranging from no symptoms to severe symptoms. ${ }^{10}$ Fonseca's anamnestic index was significantly and positively correlated with the Helkimo index with a reliability of $95 \%(r=0.6169, p<0.05)$. The present study provides information about the prevalence and severity of TMDs, based on the FAl, among the dental students of Qassim University.

Based on data collected for the present study, $55 \%$ of the students were TMD free, whereas $45 \%$ were classified as having mild, moderate, or severe TMD. These findings are consistent with those of Debora et al., ${ }^{12}$ who reported that about $40 \%$ of Brazilian college students had TMD, while about $60 \%$ had no TMD. Mild TMD was found to be more prevalent than moderate or severe TMD. However, the findings of this study differed from those of Nomura et al., ${ }^{6}$ who found that about $53 \%$ of Brazilian dental undergraduate students had symptoms of TMD.

In our study, we found that more females (52\%) in the age group of 20 to 24 years were impacted by TMD than males (42\%) of the

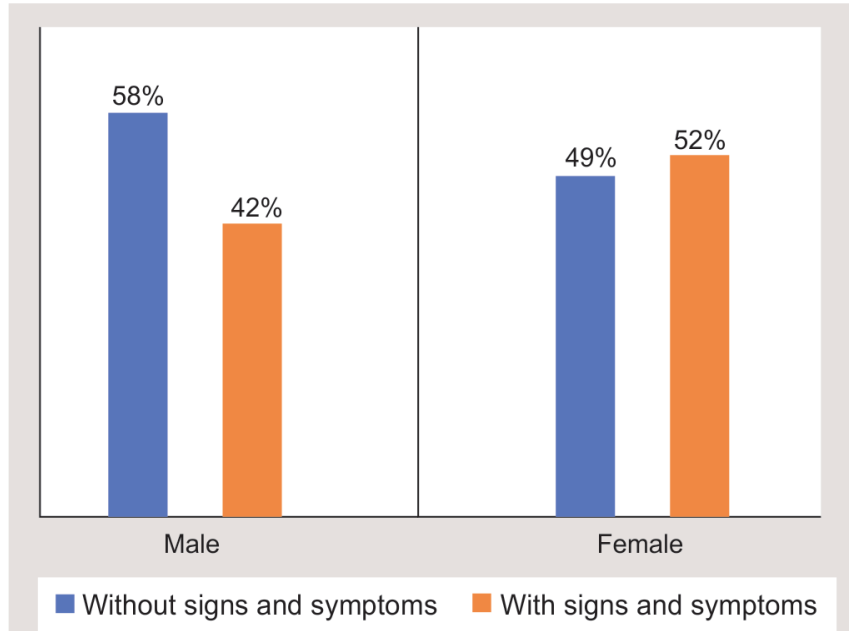

Fig. 2: Gender distribution of temporomandibular disorders

same age group. These findings indicate that the female gender is associated with increased symptoms of TMD, which is consistent with Graue et al., ${ }^{13}$ Solberg et al., ${ }^{14}$ Klineberg et al., ${ }^{15}$ and Shiau et al., ${ }^{16}$ who reported that women have a higher predisposition to TMD symptoms than men. Previous research has shown that women are more likely to develop symptoms of TMD than men due to physiological differences, including the structures of their muscles and connective tissues as well as variations in hormones. ${ }^{12,17}$ Stress is considered to be one of the most important etiological factors for temporomandibular dysfunctions, and $58 \%$ of female respondents reported experiencing stress when compared with $31 \%$ of males reporting the same. A study by Pesqueira et al. ${ }^{18}$ supports this association. Therefore, the high prevalence of TMD among medical students could be attributed to the high levels of stress and anxiety associated with engaging in the sophisticated curriculum of the various medical fields. ${ }^{19}$ Symptoms of stress observed in medical students include TMD-associated behaviors such as grinding teeth, clenching, and bruxism. These behaviors result in an overutilization of jaw muscles, leading to spasm, muscle fatigue, and even pain. ${ }^{20}$ It is difficult to measure the prevalence of anxiety and stress within a single study; thus, longitudinal research is needed to link high anxiety and stress with TMD symptoms.

Using Fonseca's questionnaire, this study has been able to show the characteristics associated with TMD severity. The symptom that received the highest rating of severity was reported to be frequent 


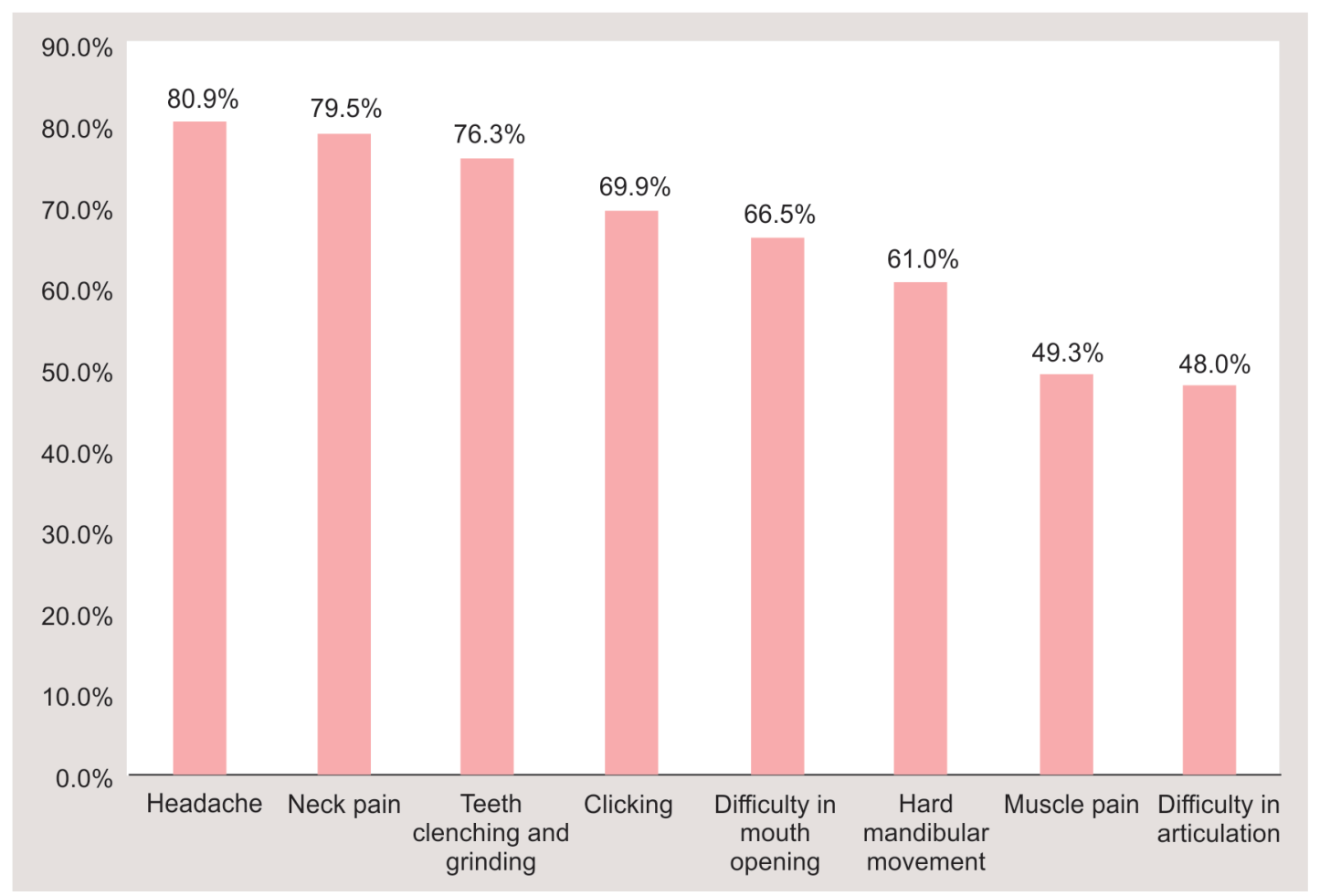

Fig. 3: Severity of temporomandibular disorders_-signs and symptoms

headaches, followed by neck pain, teeth clenching and grinding, clicking, difficulty in opening the mouth, difficulty in moving the mandible from side to side, muscle pain, and, finally, difficulty in articulating the teeth. These characteristics are good indicators for TMD and can be used in the early detection and diagnosis of the disorder. Physicians can use this information to justify the need to perform a complete clinical examination using validated modality instruments. The treatment process for TMD requires that students employ prevention measures, such as relaxation to release tension in the impacted areas.

Our study using the Fonseca's questionnaire provides information regarding the prevalence and diagnosis of TMDs in dental students, but long-term clinical studies are needed to inform methodologies for preventing the severity and ensuing complications associated with TMDs.

\section{Conclusion}

This study has successfully shown that Fonseca's questionnaire can help to identify the symptoms of TMD. The simple format of the survey elicited reports of the signs and symptoms associated with the deterioration of the TMJ or the entire stomatognathic system. The Fonseca's questionnaire was able to classify dental students into categories for mild, moderate, and severe TMD based on the acuteness of their symptoms. Future researchers should aim to conduct longitudinal studies among dental or medical students so that they can follow the change in prevalence and severity of TMD over a longer time frame.

\section{References}

1. De Leeuw R, Klasser GD. Orofacial pain: guidelines for assessment, diagnosis, and management. Am J Orthod Dentofacial Orthop 2008;134(1):171. DOI: 10.1016/j.ajodo.2008.05.001.
2. Al-Khotani A, Naimi-Akbar A, Albadawi E, et al. Prevalence of diagnosed temporomandibular disorders among Saudi Arabian children and adolescents. J Headache Pain 2016;17(1):41. DOI: 10.1186/ s10194-016-0642-9.

3. Lee JY, Kim YK, Kim SG, et al. Evaluation of Korean teenagers with temporomandibular joint disorders. J Korean Assoc Oral Maxillofac Surg 2013;39(5):231-237. DOI: 10.5125/jkaoms.2013.39.5.231.

4. Vojdani M, Bahrani F, Ghadiri P. The study of relationship between reported temporomandibular symptoms and clinical dysfunction index among university students in shiraz. Dent Res J 2012;9(2):221. DOI: 10.4103/1735-3327.95240.

5. LeResche L, Saunders K, Von Korff MR, et al. Use of exogenous hormones and risk of temporomandibular disorder pain. Pain 1997;69(1-2):153-160. DOI: 10.1016/s0304-3959(96)03230-7.

6. Nomura K, Vitti M, Oliveira AS, et al. Use of the Fonseca's questionnaire to assess the prevalence and severity of temporomandibular disorders in Brazilian dental undergraduates. Braz Dent J 2007;18(2):163-167. DOI: 10.1590/s0103-64402007000200015.

7. Bonjardim LR, Gavião MB, Pereira LJ, et al. Signs and symptoms of temporomandibular disorders in adolescents. Braz Oral Res 2005;19(2):93-98. DOI: 10.1590/s1806-83242005000200004.

8. Dhir P. Possible etiological factors and clinical features of TMD. J Adv Clin Res Insig 2016;3(3):91-93. DOI: 10.15713/ins.jcri.113.

9. Schiffman E, Ohrbach R, Truelove E, et al. Diagnostic criteria for temporomandibular disorders (DC/TMD) for clinical and research applications: recommendations of the international RDC/TMD consortium network and orofacial pain special interest group. J Oral Fac Pain Head 2014;28(1):6. DOI: 10.11607/jop.1151.

10. Campos JA, Carrascosa AC, Bonafé FS, et al. Severity of temporomandibular disorders in women: validity and reliability of the Fonseca anamnestic index. Braz Oral Res 2014;28(1):16-21. DOI: 10.1590/s1806-83242013005000026.

11. Helkimo M. Studies on function of the mastigatory system. Index for anamnestic and clinical dysfunction and occlusal state. Swed Dent J 1974;67(2):101-121.

12. Debora BG, Thaís CC, Anamaria SO, et al. Anamestic index severity and signs and symptoms of TMD. J Craniomandib Pract 2006;24:112-118. 
13. Graue AM, Jokstad A, Assmus J, et al. Prevalence among adolescents in Bergen, Western Norway, of temporomandibular disorders according to the DC/TMD criteria and examination protocol. Acta Odontol Scand 2016;74(6):449-455. DOI: 10.1080/00016357.2016.1191086.

14. Solberg WK, Woo MW, Houston JB. Prevalence of mandibular dysfunction in young adults. J Am Dent Associat 1979;98(1):25-34. DOI: 10.14219/jada.archive.1979.0008.

15. Klineberg I, McGregor N, Butt H, et al. Chronic orofacial muscle pain a new approach to diagnosis and management. Alpha Omegan 1998;91(2):25-28.

16. Shiau YY, Chang C. An epidemiological study of temporomandibular disorders in university students of Taiwan. Community Dent Oral Epidemiol 1992;20(1):43-47. DOI: 10.1111/j.1600-0528.1992. tb00672.x.
17. Celic R, Jerolimov V, Zlataric DK. Relationship of slightly limited mandibular movements to temporomandibular disorders. Braz Dent J 2004;15(2):151-154. DOI: 10.1590/s0103-64402004000200012.

18. Pesqueira AA, Zuim PR, Monteiro DR, et al. Relationship between psychological factors and symptoms of TMD in university undergraduate students. Acta Odontológica Latinoamericana 2010;23(3):182-187.

19. Ryalat S, Baqain ZH, Amin WM, et al. Prevalence of temporomandibular joint disorders among students of the university of Jordan. J Clin Med Res 2009;1(3):158. DOI: 10.4021/jocmr2009.06.1245.

20. Wahid A, Mian Fl, Razzaq A, et al. Prevalence and severity of temporomandibular disorders (TMD) in undergraduate medical students using Fonseca's questionnaire. Pakistan Oral \& Dental Journal 2014;34(1):38-41. 